\title{
Philosophiques
}

\section{Normativité, signification et acte locutionnaire}

\section{Charles Groulier}

Volume 45, numéro 2, automne 2018

URI : https://id.erudit.org/iderudit/1055269ar

DOI : https://doi.org/10.7202/1055269ar

Aller au sommaire du numéro

Éditeur(s)

Société de philosophie du Québec

ISSN

0316-2923 (imprimé)

1492-1391 (numérique)

Découvrir la revue

Citer cet article

Groulier, C. (2018). Normativité, signification et acte locutionnaire. Philosophiques, 45(2), 391-418. https://doi.org/10.7202/1055269ar

\section{Résumé de l'article}

La question de savoir si la signification est normative et comment préciser l'idée de normativité sémantique fait l'objet de nombreux débats actuels. Nous proposons de partir de l'hypothèse qu'un langage est un système de règles, et qu'apprendre un langage c'est apprendre à obéir à des règles qui régissent l'usage de ses expressions. Nous distinguons d'abord entre différentes notions de signification et de normativité. Puis nous examinons de façon critique deux objections à l'idée d'une normativité sémantique : la première réduit la normativité sémantique au « doit » instrumental, l'autre à la normativité épistémique. Nous proposons ensuite une conception alternative de la normativité sémantique fondée sur le concept (propre à la théorie des actes de langage) d'acte locutionnaire. 


\title{
Normativité, signification et acte locutionnaire
}

\author{
CHARLES GROULIER
}

École des Hautes Études en Sciences Sociales (EHESS)

groulier@ehess.fr

\begin{abstract}
RÉSUMÉ. - La question de savoir si la signification est normative et comment préciser l'idée de normativité sémantique fait l'objet de nombreux débats actuels. Nous proposons de partir de l'hypothèse qu'un langage est un système de règles, et qu'apprendre un langage c'est apprendre à obéir à des règles qui régissent l'usage de ses expressions. Nous distinguons d'abord entre différentes notions de signification et de normativité. Puis nous examinons de façon critique deux objections à l'idée d'une normativité sémantique: la première réduit la normativité sémantique au «doit» instrumental, l'autre à la normativité épistémique. Nous proposons ensuite une conception alternative de la normativité sémantique fondée sur le concept (propre à la théorie des actes de langage) d'acte locutionnaire.
\end{abstract}

\begin{abstract}
There are many ongoing debates over whether meaning is normative or about how the idea of semantic normativity is to be fleshed out. We suggest to start from the assumption that language is a system of rules, and learning a language is learning to obey the rules for the use of its expressions. We first distinguish between different notions of meaning and of normativity. Then we critically examine and reject two objections to the idea of semantic normativity: one reducing semantic normativity to instrumental "ought", the other to epistemic normativity. We then propose an alternative conception of semantic normativity based on the speech-acts theorists' concept of locutionary act.
\end{abstract}

L'idée selon laquelle parler et comprendre un langage revient à utiliser des expressions selon certaines règles fut défendue par Ludwig Wittgenstein ${ }^{1}$ et interrogée par Wilfrid Sellars². Dans son ouvrage de 1982, Règles et langage privé $^{3}$, Kripke tire de son interprétation de Wittgenstein une conception normative de la signification. Cette dernière s'articule essentiellement autour des notions de "correction", de "justification » ou de "devoir ", et introduit explicitement le terme "normatif » pour qualifier la relation entre intention, signification et usage ${ }^{4}$. L'interprétation kripkéenne a donné lieu à de foisonnantes discussions sur l'existence d'une dimension normative propre au domaine sémantique ${ }^{5}$. À partir des remarques de Wittgenstein et de son

1. L. Wittgenstein, 2004.

2. W. Sellars, I954, dans K. Sharp et R. Brandom, 2007.

3. S. Kripke, Règles et langage privé. Introduction au paradoxe de Wittgenstein, trad. T. Marchaisse, Seuil, 1996. Nous nous référerons dorénavant à la traduction.

4. Ibid., p. 49 .

5. Parmi lesquelles: C. Wright (I984), P. Boghossian (I989 et 2008), R. Brandom (2010), K. Glüer \& A. Wikforss (2009), A. Hattiangadi (2007), A. Gibbard (20I 2).

PHILOSOPHIQUES 45/2 - Automne 2018, p. 391-418 
interprétation, et en mettant entre parenthèses toute prétention exégétique, il est possible d'isoler une première formulation très générale de cette conception: appliquer un terme ou utiliser une expression linguistique revient à suivre une règle. En fonction de sa signification, il existe des manières correctes et incorrectes d'utiliser une expression, des manières dont celle-ci doit être appliquée. Tout usage, toute production linguistique, toute énonciation douée de sens est un phénomène normatif, au sens où l'on ne peut pas rendre compte de ce qu'est faire une énonciation linguistique douée de sens sans user d'un concept normatif.

Nous essaierons de clarifier cette thèse en l'opposant à différentes interprétations et objections récentes. Pour ce faire, nous suivrons le plan suivant: après avoir introduit le paradoxe sceptique de Kripke et son argument de la normativité, nous proposerons $(\mathbb{I}$ ) une distinction entre normes et règles, selon le rapport qu'elles entretiennent aux devoirs catégoriques et hypothétiques d'une part, et à la pratique et à la convention d'autre part. À partir de ce cadre conceptuel, nous évaluons et rejetons ensuite deux types d'objection à la conception normative de la signification: l'une $(\mathbb{2} 2)$ visant à réduire la normativité sémantique à une forme de normativité épistémique, l'autre $\left(\mathbb{S}_{3}\right)$ à la réduire à une forme de normativité instrumentale. Nous terminerons $(\$ 4)$ en proposant une nouvelle interprétation de la thèse de la normativité de la signification à partir du concept d'acte locutionnaire, en défendant l'idée que la normativité en jeu relève de ce concept, c'est-à-dire de la manière dont nous concevons ce qu'est un locuteur, le type d'acte qu'il accomplit au moyen du langage et la norme à laquelle il est, par définition, soumis. Notre thèse est donc "métaconceptuelle" (plutôt que métaphysique), au sens où elle ne porte pas sur la nature de la signification, sur ce qu'est la signification, mais sur certains concepts relatifs à la signification.

Commençons par rappeler rapidement la manière dont Kripke introduit l'objection de la normativité. Le sceptique mis en scène par Kripke pose un défi qu'on pourrait appeler "constitutif", lorsqu'il demande ce qui constitue le fait que je signifie bien la fonction PLUS ${ }^{6}$ lors de mon usage du terme "plus». Notre sceptique interroge la signification d'un terme mathématique (le terme "plus» ou fonction d'addition) mais la portée de sa question est tout à fait générale: elle porte sur la signification et la possibilité de signifier quoi que ce soit. Le sceptique soutient qu'aucune représentation ou image mentale ne peut constituer la signification d'un terme, dans la mesure non seulement où aucune représentation ou image ne nous dit comment utiliser le terme, mais, de plus, où toute image ou représentation peut être interprétée de façon multiple. Dans le cas de la signification et de la saisie de la signification de "plus ", l'appel à une forme d'image mentale de la fonction PLUS paraît peu éclairante et pour le moins obscure d'un point de vue

6. On suivra dorénavant un usage répandu, en utilisant l'écriture en capitales pour désigner les significations ou concepts. 
phénoménologique. Faire l'hypothèse de quelque chose comme une règle présente à l'esprit, qui sied mieux à la façon dont nous nous représentons intuitivement la signification d'un concept mathématique comme celui d'addition, ne permet néanmoins pas d'échapper au paradoxe, car toute règle peut elle aussi être réinterprétée. Faire intervenir des règles pour interpréter des signes ou d'autres règles ne fait que repousser le problème.

Kripke envisage alors l'hypothèse que le fait en vertu duquel le locuteur signifie bien PLUS par l'expression "plus » est sa disposition à répondre "I 25 " chaque fois que la question " $68+57$ » lui est posée, et de manière générale, à utiliser le terme "plus» pour appliquer la fonction d'addition (comme, en toute hypothèse, notre communauté linguistique est elle-même disposée à le faire). Mais cette hypothèse est rejetée, car elle revient à dire, comme le formule Kripke:

«I 25 » est la réponse que vous êtes disposé à donner et (peut-on ajouter) c'est aussi ce que vous auriez répondu par le passé. Parfait, je sais donc maintenant que « $\mathrm{I} 25$ " est la réponse que je suis disposé à donner (et donne ici même!), et il est peut-être utile d'apprendre - comme une donnée factuelle brute - que j'aurais répondu de même par le passé. Mais qu'est-ce qui m'indique ici que "I25" est (ou était) une réponse justifiée en termes de directives que je me serais données, et non une réponse arbitraire et injustifiée, jaillissant de moi comme un diable hors de sa boîte?

L'idée de «directive» a, semble-t-il, une portée normative, dans la mesure où ce n'est que parce qu'il existe des directives déterminées qu'une action peut être dite conforme à une directive, et ce n'est que parce que le locuteur a un accès à ces directives qu'il peut être dit lui obéir. De plus, le fait qu'un locuteur ait certaines dispositions verbales, par exemple à utiliser le mot "vache » en présence de vaches ou pour faire référence à des vaches, ne rend pas compte du fait que certains usages sont corrects et d'autres incorrects. À supposer qu'un ensemble de dispositions verbales puissent déterminer l'extension du terme "vache ", en quoi cet ensemble permet-il de comprendre le fait qu'un seul type d'usage est correct, que certains usages sont justifiés, qu'ils correspondent à la manière dont le locuteur doit utiliser le terme, que le locuteur s'est conformé à la règle? Kripke propose le diagnostic suivant:

Le dispositionnaliste nous fournit une explication descriptive de cette relation: si «plus» signifiait l'addition, alors je répondrai «I25". Mais une telle réponse n'est pas appropriée, car cette relation est essentiellement normative. La question n'est pas que je répondrais "I25", si je signifiais l'addition par "plus ", mais que, si j'entends me conformer à la signification que j'attribuais par le passé à «+», je devrais répondre «I $25 »^{8}$.

7. Kripke, I996, p. 34 .

8. Kripke, I996, p. 49. 
La conception dispositionnaliste est incapable de rendre compte de cette relation normative entre la signification et l'usage.

Comme l'a formulé Boghossian:

[L]'intuition de Kripke fut de réaliser que cette observation peut être convertie sous la forme d'une condition d'adéquation pour toute théorie de la détermination de la signification: tout candidat au titre de propriété en vertu de laquelle une expression a une signification doit être tel qu'il fonde la «normativité » de la signification - il doit être possible d'extraire de toute propriété supposément constitutive de la signification d'un mot ce qu'est l'usage correct de ce mot $^{9}$.

La normativité ainsi conçue, c'est-à-dire en tant que contrainte sur toute théorie (ou conception philosophique) de la signification, comporte trois aspects centraux. Tout d'abord, connaitre la signification d'un terme revient à avoir une forme de connaissance pratique: nous savons (avec une marge d'erreur) comment utiliser le terme, ce qu'est un usage correct du terme, ce que nous devons faire ou ne pas faire avec un terme. La signification d'un terme spécifie la manière correcte de l'appliquer, comme une règle spécifie quelles actions sont correctes (obligatoires, interdites, permises). Il s'agit de ce que nous pouvons appeler l'aspect déontique de la normativité. De plus, toute règle a une dimension procédurale, constitue une forme d'instruction quant à l'action. Lorsque nous utilisons une expression, une fois que nous avons saisi le sens d'un terme, nous sentons qu'il ne nous est pas loisible de l'utiliser arbitrairement, nous nous sentons comme déterminés, contraints ou guidés, une contrainte que nous attribuons à la signification du terme. Cette sensation de détermination n'est pas une détermination de type causal, excluant la possibilité de l'erreur. Il s'agit de la relation entre une règle et son application: la règle ne détermine pas l'application, mais l'encadre, l'instruit, la contraint. Enfin, la propriété constitutive de la signification est supposée justifier l'application du terme. La relation entre la règle et son application est aussi une relation de justification, où la signification/règle du terme justifie son application particulière (si elle est correcte). La relation entre la signification conçue comme règle et l'usage du terme ne peut donc pas être causale: une relation causale entre deux propriétés ou évènements n'est pas une relation de justification.

Une partie significative du débat contemporain ${ }^{10}$ s'est concentrée sur l'analyse des termes de "correction» et de «devoir», sur le sens qu'ont ces termes lorsqu'il est affirmé par exemple qu'une expression linguistique possède des conditions de correction, ou des conditions d'usage correct, et que la signification d'un terme spécifie la façon dont on doit l'appliquer. En

9. Boghossian, I989, réédité dans Boghossian, 2008, p. I 5.

10. Voir en particulier, le débat entre D. Whiting 2009, K. Glüer \& A. Wikforss 2015 et A. Hattiangadi 2009. 
insistant ainsi sur l'aspect de la normativité que nous avons appelé déontique, nous pouvons donc reformuler notre question de la manière suivante: si la signification d'un terme implique que l'on doive l'utiliser d'une certaine façon, ce «devoir» est-il normatif et en quel sens? Y a-t-il une forme de normativité spécifiquement sémantique?

\section{Normativité, normes et règles}

Le terme de «normativité » relève d'une terminologie technique spécifiquement philosophique, qui s'est largement imposée dans les dernières décennies ${ }^{11}$. L'usage du concept s'est généralisé, sortant de son domaine privilégié (l'éthique et la métaéthique) pour s'étendre aussi bien à l'épistémologie, à la philosophie du langage et de l'esprit, ou encore à la philosophie de l'action. En un premier sens, on peut dire d'un fait, d'une propriété, d'une action ou d'une activité qu'ils sont normatifs s'ils ne peuvent être décrits qu'à l'aide de concepts comme ceux de norme, de règle, de devoir, de standard, de justification, de correction, de raison ${ }^{12}$. Par exemple, l'acte de sanctionner juridiquement, le fait que la torture soit un mal, la propriété d'être bon, d'avoir une tenue correcte selon les règles d'un club ou d'obéir à certaines normes de rationalité peuvent être considérés comme normatifs, en ce sens. Mais le terme de normativité est aussi utilisé de façon plus restrictive, originellement dans le cadre de la métaéthique, pour caractériser des concepts, des énoncés, des propriétés ou des entités comme non descriptifs, non factuels ou encore non naturels ou non naturalisables. Dans ce contexte, est normatif ce qui jouit d'un caractère ontologiquement distinct du fait, ou du naturel (au sens de ce qui peut être l'objet des sciences naturelles) et, éventuellement, possède un statut philosophiquement problématique, tandis que le concept ou l'énoncé normatif se distingue du concept ou énoncé descriptif - considéré comme central par la tradition analytique - par sa logique ou sa sémantique.

Par delà les différents usages du terme "normatif», qu'entend-on généralement par normes et par règles? Malheureusement, il n'y a pas de consensus terminologique et philosophique sur cette question, et les philosophes utilisent souvent indifféremment les deux termes. Il est généralement admis que règles et normes possèdent deux propriétés très générales en commun. Tout d'abord, elles sont supposées posséder une certaine force, exercer une certaine contrainte sur l'agent: normes et principes moraux sont invoqués pour critiquer, blâmer, corriger l'acte, les lois possèdent une fonction sociale coercitive et répressive, les règles d'un jeu encadrent et obligent

11. Comme le montre le titre de ces différents ouvrages et entrées d'encyclopédie récents: Meaning and Normativity (Allan Gibbard, 20I2), "The Normative Status of Logic» (F. Steinberger, Stanford Encyclopedia of Philosophy, 20I6), The Normativity of Rationality (Benjamin Kiesewetter, 2017).

12. La liste n'est pas exhaustive. 
les joueurs, les standards logiques et rationnels sont utilisés pour évaluer la pensée ou le discours. De plus, les normes et les règles se distinguent des lois de la nature en ce qu'elles peuvent être enfreintes: la loi de la gravitation universelle n'est ni une norme ni une règle. La difficulté survient lorsqu'on cherche à analyser cette force contraignante des normes et des règles. Intuitivement, il est clair que certaines normes ont au moins une prétention plus grande que d'autres à exercer une force contraignante sur les agents. De ce point de vue, les normes morales ou rationnelles se démarquent assez naturellement des règles d'un jeu. On suppose souvent que les normes morales ont une prétention à exercer une autorité sur le sujet rationnel qui dépasse celles de la loi juridique ou de la convention sociale. Par opposition, les règles d'un jeu ne possèdent ni ne prétendent à posséder ce type de force que nous pouvons appeler "force normative». Essayons de clarifier cette différence intuitive entre norme et règle.

Normes et règles sont typiquement exprimées à l'aide d'un vocabulaire déontique, exprimant devoirs, obligations, interdictions et permissions. En nous inspirant de Kant, on peut distinguer le concept de devoir hypothétique, qui est conditionné aux désirs et intérêts de l'agent, du concept de devoir catégorique, qui s'impose à lui de manière inconditionnelle, et dont le domaine privilégié est celui de la normativité morale. Le «doit» hypothétique est parfois appelé «instrumental ", pour insister sur le fait qu'il figure typiquement dans la conclusion d'un raisonnement pratique, comme par exemple: (intention/prémisse) je veux rester sec, (croyance/prémisse) prendre un parapluie est un moyen pour rester sec, (devoir instrumental/conclusion) je dois prendre un parapluie. Le «doit» catégorique est celui qui correspond à ce que l'on doit faire de manière inconditionnelle ou, selon la terminologie kantienne, de manière non hypothétique, comme dans le principe: "Tu ne dois pas faire à autrui ce que tu ne voudrais pas qu'il te fasse. » Le concept d'un devoir indépendant des intentions, désirs, inclinations ou intérêts de l'agent, tel que nous l'introduisons, est intentionnellement distinct du concept de devoir moral. Autrement dit, tout devoir catégorique n'est pas, en vertu de la signification de "devoir catégorique ", un devoir moral. Il ne serait donc pas contradictoire d'affirmer qu'un devoir épistémique, comme le fait que l'on doit croire que $p$ si et seulement si $p$ est vrai, soit catégorique ${ }^{13}$.

Comme l'a remarqué P. Foot dans un article fameux ${ }^{14}$, caractériser le devoir catégorique par son inévitabilité, par le fait que sa force s'exerce même sur l'agent qui n'a pas l'intention de s'y soumettre, est insuffisant: les devoirs que stipule l'étiquette continuent de s'appliquer même à celui qui a

13. Pour une défense de la catégoricité de la norme épistémique de la croyance, voir Engel 2013.

14. Foot (I972). 
de bonnes raisons de ne pas s'y conformer. Ce n'est manifestement pas ce genre de catégoricité que certains philosophes cherchent à déterminer:

Bien que les gens donnent comme raison de faire quelque chose le fait que l'étiquette l'exige, nous ne prenons pas cette considération comme nous donnant, en elle-même, une raison d'agir. Les considérations ayant trait à l'étiquette n'ont pas le pouvoir de donner automatiquement des raisons (any automatic reason-giving force), et un homme pourrait être justifié (might be right) à nier qu'il ait des raisons de faire "ce qui se fait $»^{15}$.

Foot suggère que le genre de règles dont l'étiquette est un exemple manque d'une justification rationnelle, au sens où leur simple invocation ne constitue pas une raison. Même si on les considère comme donnant des raisons d'agir (ce que Foot ne fait pas), le fait que «c'est la règle» n'est jamais une raison suffisante, du moins dans le cas des règles qui comportent une forte dimension conventionnelle. Il faut donc ajouter une nouvelle distinction entre le «doit» catégorique et le «doit» inévitable ${ }^{16}$, au sens où il continue de s'appliquer indépendamment des désirs de l'agent.

Nous pouvons distinguer normes et règles selon le rapport qu'elles entretiennent à la force normative d'une part, et à la convention et la pratique d'autre part. Stipulons qu'une norme est une prescription ou un standard typiquement exprimé à l'aide du «doit » catégorique. La norme prétend ainsi exercer une force normative catégorique sur les agents ${ }^{17}$. Les normes constituent des raisons ou des justifications pour l'action ou la pensée. Les normes morales sont le prototype même de la norme. Par opposition, les règles peuvent être invoquées comme raison ou justification, mais ne constituent pas en elle-même (c'est-à-dire, indépendamment de toute autre considération) une raison ou une justification.

Les règles peuvent prendre des formes extraordinairement variées, mais celles qui nous intéressent en particulier sont les règles intrinsèquement liées à une activité ou pratique en partie conventionnelle (comme le langage). On appelle parfois ces règles des règles constitutives. Le prototype de la règle constitutive est la règle du jeu. Un jeu (le poker, les échecs, le football) est une pratique essentiellement constituée par des règles, qui possèdent une forte dimension conventionnelle. Selon le modèle des règles constitutives de Searle ${ }^{18}$, celles-ci prennent essentiellement la forme suivante: dans la

15. Id., p. 309.

16. Nous empruntons la terminologie à A. Hazlett (20I3, chap. IX, p. 264).

17. Nous supposons que les normes ont une "prétention" à exercer une force normative catégorique sur les agents, car nous ne voulons pas prendre parti ici sur la question de savoir s'il existe véritablement des devoirs catégoriques. Néanmoins, nous voulons conserver cette différence de force normative existant intuitivement entre les normes morales et les règles comme les règles d'un jeu ou les règles du code de la route.

18. Searle, I969. Zelaniec 20 Io précise que Czesław Znamierowski est le premier à étudier le phénomène des règles constitutives. 
pratique X, l'action de $\phi$-er compte comme l'action de $\theta$-er. Par exemple, dans le cadre du football (soccer), l'action de faire franchir la ligne des buts au ballon compte comme marquer un point. Ces règles constituent, par leur existence même, l'activité qu'elles encadrent et se distinguent ainsi des règles régulatives, qui régissent une activité préexistante. Les règles constitutives circonscrivent le champ de toute action possible, à l'intérieur du jeu. Elles stipulent les conditions nécessaires et suffisantes pour accomplir une action du jeu. En ce sens, les règles constituent le jeu.

Notons que cette distinction doit être comprise comme une distinction conceptuelle plutôt qu'ontologique ${ }^{19}$. Qu'une règle soit constitutive ou régulative dépend en partie de la manière dont on décrit l'action qui tombe sous la règle. Les lois portant sur l'héritage peuvent être considérées comme régulatives si l'on décrit l'acte qu'elles régulent comme un acte non juridique pré-existant, par exemple comme l'acte de transmettre un bien à un descendant. Mais elles sont des règles constitutives si l'acte consiste à léguer une propriété, dans la mesure où leg et propriété n'existent que relativement à un système juridique.

L'existence de règles conventionnelles est une condition nécessaire mais non suffisante de la pratique essentiellement régulée, ce que Raz appelle un «système normatif autonome ${ }^{20}$ ». Une telle pratique comporte aussi une finalité ou une valeur autonome. Pour être un jeu, une pratique régulée doit avoir pour finalité la victoire, pour valeur positive le fait de gagner et pour valeur négative le fait de perdre ${ }^{21}$. Mais le fait que tout jeu comporte ce but n'est pas plus une règle qu'une convention: ce but ne varie pas selon les différents jeux et ne figure pas dans les règles. Le but donne leur force aux règles: un agent n'est soumis aux règles d'un jeu que lorsqu'il a l'intention ou qu'il est en train de jouer, c'est-à-dire lorsqu'il cherche à gagner selon les règles. Cette définition n'interdit pas que l'on puisse jouer en trichant ou jouer avec l'intention de perdre, mais le joueur doit au moins prétendre avoir l'intention de gagner et de respecter les règles ${ }^{22}$. Toute pratique, même la plus fortement conventionnelle, ne peut pas être définie uniquement à partir d'un ensemble de règles: cet ensemble doit constituer un système, unifié autour d'un but, d'une valeur, d'une finalité propre.

19. Nous remercions un rapporteur anonyme de Philosophiques pour sa remarque sur la nécessité de cette distinction.

20. Raz, I999, chap IV.

21. Le concept de jeu est notoirement difficile à circonscrire, et il est peu probable qu'il soit possible de fournir un ensemble de conditions nécessaires et suffisantes pour qu'une activité tombe sous ce concept. Certains jeux n'ont pas vraiment de règles: nous pouvons dire que deux chats jouent ensemble, mais suivent-ils des règles? Nous nous concentrons donc ici sur le sous-ensemble des jeux qui sont régulés.

22. Raz I975, chap. IV. 
En adaptant la typologie des systèmes normatifs de $\mathrm{Raz}^{23}$, nous dirons qu'un système de règles satisfait deux conditions:

— Les règles de ce système ont une force normative conjointe: l'agent doit obéir à l'ensemble (ou à une majorité) des règles du système, l'obéissance à une règle n'a pas de sens et est inutile si l'agent ne se conforme pas aux autres. Par exemple, l'obéissance aux règles d'un jeu ou aux règles de politesse n'a de sens que si l'agent a l'intention de se conformer à une part importante de ces règles.

— Le système est articulé autour d'une valeur commune. Celle-ci rend compte de la force normative des règles, et cette valeur ne peut être décrite qu'en référence aux règles.

La valeur ici considérée n'a pas à être une valeur en soi ou une valeur ultime, comme le bien ou l'égalité. Dans le cadre d'un système de règles, règles et valeurs sont interdépendantes: la valeur donne le sens de la pratique et unifie les règles, et les règles donnent le contenu de la valeur. Dans le cas du jeu, il y a bi-valence: gagner et perdre. La valeur-victoire donne le sens de ce qui est recherché par les participants (ce qui guide leur comportement, leur stratégie) et transmet une force normative aux règles, et en même temps, ce en quoi consiste le fait de gagner dans un jeu particulier, le contenu de sa valeur, ne peut être explicité qu'à l'aide des règles. La politesse est, en ce sens, un système de règles organisé autour des valeurs "être poli/être impoli ». L'individu qui enlève son chapeau en entrant dans une pièce le fait dans le but d'être poli, et d'éviter d'être impoli. Et ce que c'est qu'être poli ne consiste en rien d'autre que de respecter les règles de la politesse. La valeur d'un système de règles est distincte de la fonction qu'il possède au sein d'une collectivité ou de la fin que se donnent les participants. Le jeu peut avoir pour fonction sociale le sentiment collectif, et le joueur peut, à titre individuel, y chercher un divertissement. De même, la politesse peut servir de moyen de cohésion sociale, et une personne particulière peut respecter les règles de politesse pour bénéficier du statut symbolique qu'il croit pouvoir en tirer. Mais les valeurs de ces systèmes ne sont pas plus le divertissement que la distinction sociale. Toutefois, la valeur du système de règles et la fin ou fonction de la pratique corrélée sont intimement mêlées: la fonction de la pratique peut donner une raison de modifier, voire d'enfreindre certaines règles. On peut chercher à modifier les règles d'un jeu, institutionnellement ou à l'occasion d'une partie, si celles-ci nuisent à la fonction divertissante du jeu. Une personne peut contrevenir de manière justifiée à une

23. Id. chap. IV. Joseph Raz parle de systèmes normatifs plutôt que de systèmes de règles parce qu'il inclut dans son concept de norme à la fois notre concept de règle (comme quelque chose d'essentiellement conventionnel et constitutif d'une pratique) et notre concept de norme (comme prescription ou standard qui possède une prétention à avoir une force normative catégorique). 
règle de politesse si elle est justifiée à penser que, dans un contexte particulier, l'obéissance à la règle peut offenser un tiers.

Les règles constitutives forment donc un ensemble solidaire d'une pratique qui est, au moins en partie, conventionnelle. Elles sont typiquement exprimées au moyen de ce que nous avons appelé le «doit» inévitable. Le jeu, les rites religieux, le sport, le shopping, la chasse, l'artisanat, l'agriculture, l'art militaire sont, en un sens très large, des pratiques, mais elles ne sont pas toutes conventionnelles, ou ne comportent pas toutes au même degré des éléments conventionnels ${ }^{24}$. Il est clair que le jeu, dont les règles constituent le prototype de la règle dans le sens que nous avons introduit, est la pratique la plus conventionnelle qui soit. Nous dirons qu'une pratique et les règles qu'elles supposent sont conventionnelles lorsqu'elles impliquent essentiellement un accord (explicite ou tacite) entre ceux qui y participent, de sorte que l'objet de l'accord (les règles) peut en principe être modifié ou annulé. Ainsi, il est conventionnel qu'au poker les joueurs possèdent chacun deux cartes.

Les normes morales et les règles du jeu constituent deux pôles prototypiques aisément identifiables dans le cadre que nous avons proposé: d'un côté, les normes, qui exercent (ou ont une prétention à exercer) une forme d'autorité non conditionnelle, de l'autre, les règles, qui possèdent une forte dimension conventionnelle et sont directement liées à des pratiques. Une difficulté survient lorsqu'on s'intéresse aux concepts de norme et de règle appliqués à d'autres domaines: certains pans de notre activité mentale (nos états, processus ou actes mentaux comme les croyances, les décisions, les intentions ou les raisonnements) sont-ils soumis à des normes ? ${ }^{25}$ Ces normes impliquent-elles des devoirs catégoriques ou hypothétiques? L'activité linguistique est-elle constituée par des règles? En particulier, en quel sens peuton dire que l'on doit utiliser un terme conformément à sa signification, comme le suppose Kripke lorsqu'il affirme que la relation entre la signification et l'usage est normative, et non descriptive? Si l'usage des mots est encadré par des règles constitutives du langage, le langage lui-même est-il articulé autour de quelque chose d'analogue à la valeur, au sens de Raz?

\section{La norme de l'assertion}

Une première réponse consiste tout simplement à dire que la relation normative entre la signification et l'usage se réduit à celle du devoir hypothétique, car le locuteur ne doit utiliser un terme d'une certaine manière que pour autant qu'il a une certaine intention — l'intention d'utiliser le terme selon sa

24. Il n'est pas toujours évident de savoir si une pratique comporte essentiellement un élément conventionnel. Par exemple, la pratique du shopping n'est certainement pas une pratique conventionnelle comme le jeu, mais elle comporte un élément conventionnel: la monnaie. Toutefois cet élément est-il essentiel?

25. Cf. Engel $20{ }_{3} 3$ sur la norme de la croyance. 
signification conventionnelle ou l'intention de communiquer efficacement, etc. Avant de nous pencher sur cette idée en détail, envisageons d'abord une autre hypothèse. On peut tenir pour acquis le fait que la relation entre la signification et l'usage relève d'un type de devoir instrumental, dans la mesure où elle repose sur une intention du locuteur, et néanmoins préserver une dimension normative plus forte. Une suggestion possible serait d'expliquer la force normative du "doit» qui apparaît dans des énoncés comme "on doit utiliser “+” pour signifier la fonction d'addition» à partir de la notion de vérité. Par exemple, Daniel Whiting ${ }^{26}$ propose ainsi de réduire la normativité sémantique à la norme constitutive de l'assertion, qui n'est autre que la vérité. Mais en quoi le fait d'asserter le vrai n'est-il pas soumis à l'intention du locuteur? Nous pouvons, bien entendu, ne pas vouloir dire la vérité, ou avoir des raisons de ne pas dire la vérité, mais il n'en reste pas moins que l'acte illocutionnaire qu'est l'assertion est constitué par une norme de vérité, au sens où l'on doit, prima facie, s'efforcer d'asserter le vrai. Après tout, un menteur aurait quelques difficultés à transmettre une information fausse et à tromper son auditeur s'il n'existait pas une pratique consistant essentiellement (ou constitutivement) à dire la vérité, c'est-à-dire à asserter ${ }^{27}$.

Partons d'une formulation possible de l'idée selon laquelle les expressions linguistiques ont des conditions de correction: "si "vert" signifie VERT, alors il est correct d'appliquer "vert" à un objet $x$ si et seulement si $x$ est vert». Faire une application correcte revient alors à faire une application vraie, ce qui n'est rien d'autre que d'asserter correctement. Appliquer "vert" à l'herbe, c'est asserter que l'herbe est verte, et asserter que l'herbe est verte, c'est appliquer "vert" à l'herbe. Or l'assertion est, selon Whiting, un acte soumis à une norme de vérité, de sorte qu'une assertion est correcte si et seulement si elle est vraie ${ }^{28}$. Ainsi, lorsqu'un locuteur applique "vert» à la neige, il fait non seulement une assertion incorrecte, mais il viole aussi une norme sémantique. La normativité de la signification dérive de celle de l'assertion: «Le fait qu'une expression possède une certaine signification implique qu'il existe des standards pour son application correcte ou incorrecte, et donc pour la manière dont elle doit ou peut être utilisée dans l'assertion, où la correction et l'incorrection coïncident avec, respectivement, la vérité et la faussetée ${ }^{29}$.»

Mais en quel sens la normativité de la signification peut-elle dériver de la normativité de l'assertion? Le fait qu'une assertion est correcte dérive du fait que la proposition qu'elle exprime est vraie (c'est ce qu'est supposée être

26. Whiting (20I6).

27. Thomson (2008, p. I03).

28. Whiting formule la norme en termes d'interdiction: Pour tout $S, x: S$ ne doit pas (asserter que $\mathrm{x}$ est vert) si et seulement si il est faux que $\mathrm{x}$ est vert.

29. Whiting (2016). 
une assertion), et le fait que la proposition est vraie dérive (compositionnellement) de ses constituants et de l'état du monde. Mais les significations, les contenus ou les propositions, ne sont pas supposés être vrais ou supposés être utilisés dans l'assertion, en tout cas pas plus que dans n'importe quel autre type d'acte illocutionnaire. Peut-être l'assertion doit-elle être considérée comme l'acte de langage primitif, mais de quel point de vue? Logique, pragmatique, évolutionniste ${ }^{30}$ ?

De plus, si la normativité de la signification dérive de la norme de l'assertion, c'est-à-dire d'une norme de vérité, on devrait établir l'existence, pour former une conception complète de l'activité linguistique, d'une norme différente pour chaque type d'acte illocutionnaire. Mais quelles sont les conditions de correction des questions, des ordres, des déclarations? Même si l'on déterminait les différentes normes spécifiques aux différents types d'actes illocutionnaires, il n'y aurait plus de raison de parler d'une forme unique de normativité propre à la signification dans toute sa généralité. Il faut souligner que la thèse de la normativité de la signification est générale en un double sens. D'une part, la thèse est générale en tant qu'elle porte sur toute expression indépendamment du type d'acte illocutionnaire accompli par leur moyen. D'autre part, elle est générale en tant qu'elle porte sur tous les types d'expression possédant une signification, et non sur toutes les expressions possédant une signification vériconditionnelle, c'est-à-dire les expressions qui jouent un rôle dans la détermination des conditions de vérité d'une phrase. Il est tentant de se concentrer exclusivement sur des expressions sémantiquement interprétées comme référentielles, au sens où elles réfèrent à des objets (des entités spatio-temporelles comme des vaches ou peut-être même des abstraits comme des fonctions mathématiques) et permettent de générer des conditions de vérité à partir des énoncés dans lesquels elles figurent. C'est exclusivement dans ce cadre que l'on peut identifier usage correct et application vraie, énonciation linguistiquement correcte et assertion vraie.

Le problème est qu'il existe un grand nombre d'autres expressions qui résistent à ce traitement, qui possèdent néanmoins une signification (au sens pré-théorique du terme) et au sujet desquelles la question se pose aussi de savoir ce qui constitue leur usage correct. Les quantificateurs ("tous", «quelque») ou les connecteurs («et», «ou») ne sont pas référentiels mais peuvent recevoir une interprétation dans une sémantique vériconditionnelle. Toutefois, certaines expressions, qui intéressent naturellement plus les pragmaticiens que les sémanticiens, et généralement peu les philosophes du langage, sont telles qu'elles ne participent pas au contenu vériconditionnel des

30. En ce qui concerne l'hypothèse évolutionniste, Terrence Deacon (I998) insiste sur l'importance du contrat oral dans l'émergence du langage (cf. Knight, Studdert-Kennedy, Hurford I985), c'est-à-dire du genre d'acte de langage que Searle et Vanderveken (I985) appellent l'acte commissif. 
énoncés dans lesquels elles apparaissent ${ }^{31}$ : les marqueurs de discours ou connecteurs non logiques ("mais», "donc»), les adverbes illocutionnaires ou attitudinaux ("franchement», «heureusement»), les particules illocutionnaires et interjections ( «hein", " hélas », «oh»), les explétifs, les modes (interrogatifs, optatif), les expressions de présupposition, les particules d'attentions ${ }^{32}$.

La thèse de la normativité de la signification est qu'utiliser une expression correctement revient à l'utiliser selon sa signification, et non qu'appliquer une expression correctement revient à faire une assertion vraie. Nous voulons conserver la portée de la thèse: le fait qu'elle porte sur la signification en général, sur la signification de tous les types d'expression.

\section{La normativité instrumentale}

On pourrait penser que la raison pour laquelle, en reprenant l'exemple de Kripke, on doit répondre "I 25 " à la question " $68+57$ ?", si l'on entend utiliser le terme "plus » conformément à sa signification, ou que, de manière générale, on doit utiliser un terme selon sa signification, est simplement que tout agent est engagé à utiliser les moyens nécessaires en vue de la fin qu'il s'est donnée. Le locuteur doit utiliser les termes du langage selon leur signification dans la mesure où il a une certaine intention, par exemple celle de communiquer un certain contenu, et que cette intention ne peut être réalisée qu'à condition qu'il se conforme à une règle ou à un usage sémantique déterminé. Ce devoir ou cette obligation ne s'impose à lui, n'exerce une pression normative sur lui que parce que le locuteur a une certaine intention. En ce sens, il n'y a rien de plus dans ce "doit» que le genre de normativité qui conclut un raisonnement pratique ordinaire, comme par exemple: i) je veux sortir sous la pluie sans être mouillé; ii) prendre un parapluie est indispensable pour rester sec; iii) donc, je dois prendre un parapluie ${ }^{33}$. Face à cet exemple, nous pouvons faire deux remarques. Tout d'abord, le «devoir» exprimé dans la conclusion du raisonnement pratique n'a de force que conditionnelle, il est dépendant du fait que l'agent a une certaine intention. Il s'agit donc du type de devoir que nous avons appelé, suivant un usage répandu, un devoir «instrumental» (ou hypothétique). Il est donc distinct du devoir catégorique, dont la force normative est indépendante des intentions, désirs ou intérêts de l'agent. De plus, il est clair que le fait que l'agent

31. Cf. par exemple: Recanati (2004), Blakemore (2004), Iten (2005).

32. Nous reprenons le système classificatoire de Iten (2005); il ne semble pas qu'une terminologie fasse consensus. Un autre moyen de traiter ces types d'expressions est proposé par la théorie de la pertinence à travers la catégorie des significations procédurales, bien que la classe des expressions non vériconditionnelles et celle des expressions qui encodent des significations procédurales n'aient pas la même extension (Wilson et Sperber, 20I2, chap. VII, p. I 58). Les expressions procédurales (comme «donc» ou «après tout») n'encodent pas de concepts, mais guident le processus inférentiel de compréhension.

33. Verheggen 20I I propose une analyse de cette analogie et en récuse la pertinence. 
doive prendre un parapluie n'a rien à voir avec une quelconque normativité de la météo, ou n'est pas le signe que nous ayons ici affaire à un domaine spécifique de normativité. Par conséquent, le défenseur d'une telle interprétation pourra conclure les deux thèses suivantes (ou l'une des deux): i) il n'existe pas de normativité proprement sémantique ou linguistique; l'analyse révèle que ne s'exprime dans ce prétendu «devoir» rien de plus qu'une forme de normativité instrumentale, que traduit le principe selon lequel on doit employer les moyens en vue de la fin; ii) le fait que l'on doive utiliser un terme selon sa signification étant soumis à condition et dépendant d'une intention, il ne s'agit pas d'une authentique forme de normativité, c'est-àdire de la normativité intéressante et problématique du point de vue philosophique et dont l'archétype est la normativité morale ${ }^{34}$.

Soulignons que, même si cette argumentation est correcte, elle n'a aucune implication concernant les rapports entre significations et règles. Si la conclusion est que le «doit» dans l'énoncé «on doit utiliser l'expression $x$ de telle façon" n'est pas celui du devoir catégorique, de sorte qu'il n'a aucune force face à des considérations éthiques, elle est juste mais triviale. $\mathrm{Si}$ la conclusion est que ce «doit» est celui du devoir hypothétique, de l'engagement à l'égard de nos intentions, ou de l'expression de la rationalité pratique, cela n'implique aucunement que signifier ne consiste pas à suivre une règle, que les significations conventionnelles ne soient pas des règles, ou que l'activité linguistique ne soit essentiellement constituée que par un système de règles.

En effet, l'infraction à la règle continue d'être une infraction même si l'agent a de bonnes raisons de contrevenir à la règle ou n'a pas l'intention de suivre la règle. Lorsqu'un agent n'a pas employé les moyens en vue de sa fin, comme dans le cas de celui qui ne prend pas son parapluie, nous ne dirons pas que son action est incorrecte ou qu'il contrevient à une règle, quoique l'on puisse dire qu'il n'a pas fait ce qu'il devait faire. Les règles sémantiques, les règles qui déterminent la manière dont un terme doit être utilisé constituent un système de règles, qui implique des devoirs et des formes de correction ou d'incorrection, indépendants des intentions particulières. Les catégories de devoir catégorique et hypothétique échouent à rendre compte de la distinction entre le fait qu'un acte doit être accompli uniquement en vertu de l'intention de l'agent, et une action qui doit être accomplie dans le cadre d'un système de règles.

34. Ces deux thèses ont la même conséquence relativement à notre problématique. D'une part, si la seule normativité est la normativité catégorique, et si les normes ou règles sémantiques ne sont pas catégoriques, alors il n'y a pas lieu de parler de normativité sémantique. D'autre part, s'il existe bien une forme de normativité instrumentale, et si c'est celle dont relèvent les prétendus devoirs sémantiques, alors il n'y a pas lieu de parler de normativité sémantique. On retrouve l'argument plus ou moins articulé ainsi chez Hattiangadi 2007, Whiting 2016 et Dretske 2000. 
Toutefois, les règles sémantiques sont, en un sens, dépendantes et "générées » par des intentions ou des activités intentionnelles. D’une part, les signes linguistiques sont conventionnels au sens où ils sont arbitraires - le signe n'a, du point de vue de sa forme, aucune similarité avec ce dont il est un signe et est donc, en principe, librement institué par les locuteurs ${ }^{35}$. D'autre part, ils sont conventionnels au sens où ils remplissent une certaine fonction de coordination (communicationnelle) entre les membres d'une communauté, impliquant une reconnaissance de cette fonction ${ }^{36}$, ou au sens où les formes linguistiques se perpétuent dans la communauté linguistique en vertu du poids du précédent, de l'habitude ou de la coutume, plutôt que grâce à un avantage intrinsèque qu'elles pourraient posséder relativement à l'accomplissement de leur fonction ${ }^{37}$.

Dans la mesure où ces règles sont conventionnelles, elles dépendent des intentions des agents impliqués dans la pratique. La signification d'une flèche indiquant la direction de Chicago dépend de notre pratique de l'interpréter ainsi. Si l'on adopte une perspective téléologique, on dira que c'est notre intention de considérer la flèche comme indiquant cette direction qui lui octroie une certaine fonction, qui fait qu'indiquer une certaine direction est ce qu'est supposé faire la flèche. Les signes artificiels comme des symboles ou des mots ne sont pas des règles ou ne sont pas supposés accomplir quoi que ce soit indépendamment de nos pratiques intentionnelles.

Pour autant, ces règles conventionnelles ne sont pas "générées" par des intentions particulières au sens où le fait que Jacques doit prendre un parapluie est généré par son intention de rester sec. Le fait que l'expression "parapluie» est utilisée pour désigner un accessoire qui protège de la pluie ne dépend pas de l'intention de Jacques. La plupart du temps, les significations conventionnelles ne dépendent pas d'une intention particulière. Peutêtre Jacques pourrait-il inventer une expression "phi » et se mettre à l'utiliser dans certaines circonstances, de sorte qu'à partir de sa pratique à l'endroit de "phi », "phi » en viendrait à avoir une certaine signification conventionnelle dans l'idiolecte de Jacques, ce que Grice appelle une signification établie ${ }^{38}$. En ce sens, la signification de «phi » dépend des intentions de Jacques: son intention d'utiliser " phi » dans telles et telles circonstances, pour exprimer tel contenu mental. Notons que, selon ce modèle, la signification établie de "phi» est dépendante non seulement de ses intentions, mais aussi de la satisfaction de ces intentions. Les circonstances d'usage de «phi» et/ou les états mentaux exprimés lors de ces usages doivent avoir suffisamment en commun pour constituer un type, de sorte qu'il y ait un sens à parler de la significa-

35. Ce qui ne signifie pas qu'en pratique, l'existence d'une forme d'expression linguistique soit le résultat d'une décision.

36. Lewis 1969.

37. Millikan 2005 , chap. I.

38. "Utterer's meaning, Sentence-meaning, Word-meaning”, rééd. dans Grice I99I. 
tion littérale de son énonciation: ce que signifie une énonciation "phi» en vertu de sa signification établie et du contexte, qui peut être distinct de $c e$ qu'a voulu dire Jacques (ce que Grice appelle la «signification du locuteur»). Si Jacques utilise "phi » complètement arbitrairement, "phi» ne peut pas avoir de signification établie ${ }^{39}$. Par conséquent, Jacques doit se rapporter à son emploi de "phi» en tant que type d'acte essentiellement soumis à certaines contraintes, un type d'acte qui peut, essentiellement, être accompli correctement ou incorrectement. Et ces contraintes n'ont, même dans le cas de la signification établie d'une expression, rien à voir avec le fait que, par ailleurs, il se peut que Jacques doive employer les moyens qu'il juge nécessaires en vue de sa fin.

À l'échelle de la communauté linguistique, on peut dire que les locuteurs ont une intention collective ou un intérêt commun à participer à une convention linguistique particulière: celle qui consiste, par exemple, dans le cas des francophones, à utiliser le mot "vache» pour désigner une sousespèce des bovidés. Il n'y a aucune obligation d'utiliser ce terme ainsi en dehors de cette pratique conventionnelle: c'est précisément en cela que l'usage linguistique est conventionnel. Mais cela ne signifie pas qu'un locuteur est libre d'utiliser un terme comme il l'entend. Le fait qu'un terme possède une signification conventionnelle ne dépend pas de l'intention d'un locuteur particulier, mais d'une pratique ${ }^{40}$, de l'inclination linguistique de la communauté, voire de l'intention d'une autorité lexicographique. Bien sûr, tout agent peut regarder n'importe quel système de règles comme un moyen en vue de son intention, de même le locuteur peut regarder la convention comme un moyen en vue de son intention d'exprimer un certain contenu, de communiquer une pensée ou d'ordonner quelque chose. Et c'est bien ce que nous faisons lorsque nous utilisons un langage: nous nous conformons aux règles (sémantiques, syntaxiques, phonologiques, etc.) dans le but d'accomplir des actes de communication, et non simplement de respecter les règles pour elles-mêmes. Mais le fait que ces règles conventionnelles servent nos intentions individuelles ou nos intérêts communs n'exclue pas que le locuteur doive se conformer à la règle, indépendamment de son intention, dans la mesure où l'existence de la règle, en tant qu'elle est conventionnelle, dépend du comportement linguistique des locuteurs. En effet, l'existence et la possibilité même des règles et de la pratique linguistique commune

39. Nous laissons de côté la thèse attribuée à Wittgenstein, selon laquelle un individu isolé ne peut pas fixer seul les conditions de correction d'une expression, puisqu'il ne peut pas distinguer entre ce qui est correct et ce qui lui semble correct (voir la note 39).

40. Précisons qu'il est ici affirmé que l'association d'un mot et d'une signification conventionnelle implique nécessairement une pratique, et, de manière contingente, une pratique sociale. Nous restons neutres quant à la thèse (plus forte) selon laquelle la signification est intrinsèquement ou nécessairement sociale (inter-subjective). Cette dernière thèse est attribuée à Witggenstein dans Kripke (I996) et cette interprétation est contestée dans Horwich (20II) et Baker \& Hacker (2009). 
dépendent des régularités que manifestent les locuteurs et de leurs efforts pour parvenir à maintenir une convention.

On pourrait rétorquer que l'existence d'un ensemble de règles circonscrites à un domaine spécifique (comme le langage, l'étiquette ou le jeu d'échecs) n'implique pas que les formes d'obligation qu'il comporte aient une force normative indépendante, et ainsi puisse soutenir que les règles spécifiques à un domaine n'ont de force que pour autant qu'elles sont appuyées et soutenues par un devoir ou une raison authentiquement normative: nous n'avons le devoir d'obéir aux règles du langage, de l'étiquette ou du jeu d'échecs que dans la mesure où celles-ci dérivent d'un devoir inconditionnel comme le devoir moral ou, peut-être, comme une norme de rationalité instrumentale, selon laquelle tout agent est engagé à ou doit employer les moyens qu'il juge nécessaires à la réalisation de son intention. Dans cette perspective, les normes ou les devoirs authentiquement normatifs sont ceux qui s'appliquent à l'agent en tant qu'agent rationnel ou en tant que sujet moral.

Cette objection suppose de restreindre le qualificatif de "normatif» au type de devoirs typiquement exprimés au moyen du «doit» catégorique. Il s'agit, nous semble-t-il, d'une question plus terminologique qu'autre chose. Ce qui, en revanche, n'est pas simplement verbal, mais véritablement substantiel, est la question de savoir si le type de devoir exprimé par des phrases comme "on doit utiliser l'expression “+” pour signifier l'addition" est hypothétique ou instrumental. À cette question nous répondons par la négative. Essayons maintenant de spécifier le type de devoir auquel nous avons ici affaire.

\section{La norme de l'acte locutionnaire}

Nous voulons pouvoir parler d'une correction (ou incorrection) sémantique (au sens de l'usage de l'expression conformément à sa signification) qui ne se réduise pas à la vérité de l'assertion, à la réussite d'un acte de communication ou à la satisfaction de l'intention particulière d'un locuteur. Le genre de correction qui nous intéresse est celle qui relève, selon la terminologie d'Austin ${ }^{41}$, de l'acte locutionnaire. Ce dernier propose une division ternaire des types d'actes qui peuvent être accomplis lors d'un acte de langage. L'acte locutionnaire consiste à utiliser des mots avec un certain sens. L'acte illocutionnaire est l'acte par lequel une locution, en tant que produit d'un acte locutionnaire, en vient à posséder une certaine force (déclarative, interrogative, etc.). L'acte perlocutionnaire consiste à produire certains effets chez l'auditeur par le moyen d'une énonciation. Notre proposition est la suivante: l'acte locutionnaire est défini, comme l'acte illocutionnaire, par des conditions de correction. Dans le cas de l'acte illocutionnaire, un acte assertif 
est correct si et seulement si le locuteur est dans la relation épistémique appropriée à son contenu, une promesse est correcte si et seulement si le locuteur contracte une forme d'engagement, etc. ${ }^{42}$. L'acte locutionnaire ne se subdivisant pas en différents types, il ne possède qu'un seul type de conditions de correction: il est correct si et seulement si le locuteur a utilisé les mots selon leur signification. Les conditions de correction de l'acte locutionnaire constituent une norme: on doit utiliser les mots selon leur signification; ou un standard évaluatif: un acte locutionnaire est correctement accompli si et seulement si le locuteur a utilisé les mots selon leur signification. Mais à quel genre de normativité avons-nous affaire, s'il ne s'agit pas d'une normativité catégorique?

La norme de l'acte locutionnaire joue, dans le cadre de l'activité linguistique, le même rôle que joue la victoire comme but ou valeur dans le système de règles qu'est le jeu. La norme de l'acte locutionnaire est ce à quoi s'efforcent d'obéir les participants à toute pratique linguistique commune. Participer à une pratique linguistique revient à "jouer selon les règles ", et les locuteurs doivent considérer comme effectives les règles conventionnelles fixées par la communauté. Ce qu'est un acte locutionnaire réussi n'est intelligible qu'en référence aux règles, de la même manière que gagner dans un jeu implique de comprendre ce en quoi consiste le fait de gagner selon les règles $d u$ jeu. L'acte locutionnaire réussi constitue ainsi le but ou la valeur intrinsèque dans le système de règles autonomes qu'est la pratique linguistique. Et ce but ou cette valeur n'est évidemment pas une valeur en soi ou une valeur ultime, pas plus que ce but ou cette valeur intrinsèque ne correspond à la fonction du langage. Le standard que constitue l'acte locutionnaire réussi ne donne pas plus la fonction du langage dans l'ordre des activités humaines que le fait de gagner n'explique la fonction du jeu dans nos sociétés.

La norme est, de plus, constitutive de l'acte. Tout locuteur est soumis à la norme dès lors qu'il s'engage dans l'acte en question. Et tout locuteur, pour compter comme un locuteur, doit être sensible à cette norme et au moins prétendre s'efforcer de la satisfaire, comme tout joueur doit au moins prétendre essayer de gagner. La norme constitutive est donc essentiellement descriptive et prescriptive: elle décrit ce en quoi consiste le fait d'accomplir un certain acte ou d'être engagé dans l'activité en question, et elle a force de prescription pour l'agent. La norme constitutive possède une dimension hypothétique ou instrumentale au sens où l'agent est libre de s'engager dans

42. Notons qu'il est possible de distinguer entre les conditions de correction et les conditions de réussite de l'acte illocutionnaire. Dans le cas de la promesse, on peut ainsi distinguer entre le fait que la promesse est sincère (le locuteur contracte un certain engagement) et le fait qu'elle soit tenue. Dans le cas de l'assertion, on peut distinguer entre le fait que le locuteur est justifié à croire le contenu asserté, et le fait que ce contenu est vrai. Nous remercions un rapporteur anonyme de Philosophiques pour avoir rappelé l'importance de cette distinction. 
l'acte ou l'activité en question, mais non au sens où la norme ne découle que de ses intentions. Elle n'est donc pas catégorique au sens où elle s'appliquerait à tout sujet indépendamment de ses désirs ou de ses intérêts, comme un devoir moral. De plus, il est clair que l'accomplissement du devoir moral prévaut sur le respect des normes linguistiques. Toutefois, la norme de l'acte locutionnaire n'est pas simplement une règle constitutive, puisqu'elle unifie l'ensemble des règles de la pratique linguistique et en constitue le but ou la valeur intrinsèque.

Remarquons qu'ainsi formulée la norme de l'acte locutionnaire semble avoir pour conséquence de rendre incorrect un usage non littéral du langage, que nous considérons pourtant ordinairement comme tout à fait correct ${ }^{43}$. En effet, si utiliser correctement une expression revient à l'utiliser selon sa signification, et si la signification d'une expression-type est la signification qu'elle possède en vertu des conventions linguistiques (la signification conventionnelle), il s'ensuit qu'il est incorrect de faire un usage non littéral d'une expression. La signification littérale d'une énonciation est la signification qu'elle possède en vertu de la signification conventionnelle des expressions qui la composent (et, si la phrase contient des expressions indexicales comme «je» ou "maintenant», en vertu des éléments du contexte d'énonciation nécessaires à la détermination du contenu de ces expressions). Par conséquent, lorsque nous utilisons métaphoriquement un terme en disant, par exemple, que "cet homme est un roc", l'expression "roc» n'est pas utilisée selon sa signification conventionnelle, et l'acte locutionnaire est incorrect. C'est une conséquence fâcheuse de la norme si celle-ci doit exclure la possibilité de l'usage non littéral.

Une réponse adéquate à ce problème supposerait l'élaboration d'une théorie de la littéralité, ce qui va bien au-delà de notre propos. Néanmoins, nous pouvons esquisser un début de réponse. Pour échapper au problème soulevé, il faut rappeler que la norme en question ne porte pas sur ce qu'un locuteur doit signifier ou communiquer au moyen du langage, mais uniquement sur la manière dont il doit utiliser une expression, compte tenu de ce que le locuteur veut communiquer. En adoptant une conception gricienne, nous pouvons distinguer entre ce qui est dit par une énonciation et ce qui est impliqué par elle. Lorsqu'un locuteur énonce "cet homme est un roc", ce qui est dit, la signification littérale de son énonciation est que cet homme est un roc, ce qui est faux. Mais ce qui est impliqué est, par exemple, que cet homme est le genre de personne sur qui on peut s'appuyer. Compte tenu du fait que le locuteur veut communiquer métaphoriquement l'idée que cet homme est le genre de personne sur qui on peut s'appuyer, il faut qu'il utilise littéralement une expression dont la signification conventionnelle permet à l'auditeur d'inférer ce qui est impliqué par l'énonciation. Or, ce dernier ne

43. Nous remercions un rapporteur anonyme de Philosophiques pour avoir soulevé ce problème. 
peut inférer l'idée que cet homme est le genre de personne sur qui on peut s'appuyer que parce que le locuteur a utilisé l'expression « roc » selon sa signification conventionnelle, c'est-à-dire pour désigner un grand bloc de pierre particulièrement solide. L'obéissance à la norme de l'acte locutionnaire n'interdit donc pas l'usage non littéral, mais au contraire le rend possible.

Si l'on accepte ce cadre théorique, la question devient donc: quelle est la fonction théorique de notre concept normatif d'acte locutionnaire? Et quel rôle explicatif est supposé jouer la norme en question dans notre conception philosophique de la signification?

Le concept d'acte locutionnaire a principalement deux fonctions, déterminées relativement à des problématiques différentes et aux réponses théoriques à ces problématiques: l'une de ces fonctions relève de la théorie des actes de langage et l'autre plus généralement de la métasémantique ${ }^{44}$, au sens de la théorie des propriétés ou faits constituant la signification. Le concept d'acte locutionnaire, dans le cadre de la théorie des actes de langage, identifie l'acte par lequel un locuteur fait une énonciation signifiante intentionnellement, en le distinguant, d'une part, de l'acte d'énonciation, et de l'autre, des actes illocutionnaires et perlocutionnaires. L'acte d'énonciation, que W. Alston ${ }^{45}$ appelle "acte sententiel», est l'acte par lequel un locuteur énonce une suite de mots formée conformément aux règles morphologiques, phonologiques et syntaxiques de la langue, considérée indépendamment de toute propriété sémantique. Il est possible d'accomplir un acte sententiel sans accomplir du même coup un acte locutionnaire, par exemple en produisant une énonciation pour s'éclaircir la voix, pour tester un microphone ou pour exercer sa prononciation. L'acte locutionnaire se distingue, d'autre part, de l'acte illocutionnaire, qui implique qu'une certaine force est attribuée à la locution. Par exemple, pour reprendre l'exemple d'Austin, un locuteur peut, face à un taureau, énoncer les mots suivants: "il va charger». Cette énonciation permet d'accomplir différents types d'actes illocutionnaires, comme une constatation ou un avertissement. Bien que, dans la pratique linguistique ordinaire, ce soit l'acte de langage en tant qu'acte illocutionnaire qui nous importe la plupart du temps, et non les modalités de sa réalisation, il ne s'ensuit pas que l'acte locutionnaire n'ait pas une forme d'existence indépendante. D'une part, il est conceptuellement pertinent de distinguer l'acte locutionnaire de l'acte illocutionnaire, puisqu'un même acte locutionnaire peut servir à accomplir différents types d'actes illocutionnaires ou perlocutionnaires. D'autre part, dans certains cas, la force illocutionnaire ordinairement attachée à tout acte locutionnaire

44. La distinction entre une théorie de la signification ou sémantique conçue comme une théorie associant de manière systématique des conditions de vérité à des énoncés et une métasémantique au sens de métaphysique de la signification est analysée dans A. Burgess et B. Sherman (20I4).

45. Alston, 2000. 
est, en quelque sorte, désactivée. Par exemple, en contexte pédagogique, l'élève est invité à produire des énonciations signifiantes, qui peuvent relever de modes grammaticaux divers (affirmatif, interrogatif, impératif), mais qui ne sont pas des actes illocutionnaires - l'écolier n'est pas réellement en train d'ordonner lorsqu'il donne un exemple d'ordre sous forme impérative ${ }^{46}$. Dans ce contexte, exprimer un contenu en respectant les règles sémantiques (et, a fortiori, syntaxiques, phonologiques) constitue la finalité même de l'acte. L'artificialité du contexte permet au locuteur d'entretenir un rapport tout à fait atypique au langage, où celui-ci cesse d'être un moyen relativement aux fins typiques des locuteurs (donner ou demander une information, exprimer un sentiment, amener quelqu'un à faire quelque chose, contracter un engagement, etc.). L'acte locutionnaire peut donc, dans certains cas, être un acte à part entière, en plus d'être, en contexte ordinaire, le composant d'un acte complexe de communication.

L'autre rôle du concept d'acte locutionnaire intervient dans le cadre plus général de la métaphysique de la signification et entre directement en jeu dans la question de la normativité de la signification. Le concept est utile pour répondre à la question suivante: qu'est-ce qui distingue un comportement verbal qui s'avère simplement être conforme à une règle du comportement verbal en tant qu'obéissance à la règle ? ${ }^{47}$ Rappelons que notre hypothèse de départ est que faire une énonciation douée de sens revient à utiliser un terme en accord avec certaines règles, de sorte que l'usage est contraint par la règle et, en ce sens, non arbitraire. Qu'est-ce qui distingue alors l'énonciation accomplie par un authentique locuteur, c'est-àdire l'acte locutionnaire, d'une énonciation qui n'est pas la manifestation d'une authentique compétence linguistique, comme celle que peut faire, après un certain conditionnement, un très jeune enfant ou un animal?

46. Il faut ajouter que le type d'acte illocutionnaire qu'il est possible d'accomplir au moyen d'une phrase peut être déterminé par la signification conventionnelle de la phrase (la signification-type que la phrase possède en vertu des significations conventionnelles de ses expressions constituantes), au moyen d'un verbe relevant d'une classe d'actes illocutionnaires, comme dans le cas des performatifs explicites suivants: "je t'ordonne de partir", «je vous déclare mari et femme ", "je promets de venir ». Il est difficile d'imaginer des cas où le locuteur accomplit un acte illocutionnaire qui ne correspond pas au type d'actes que les expressions "ordonner ", "déclarer» ou "promettre » rendent possible. Il semble donc que, dans le cas des expressions désignant des types d'actes illocutionnaires, connaître la signification conventionnelle implique de savoir quel genre d'actes illocutionnaires ces expressions permettent d'accomplir. Il ne s'ensuit pas néanmoins que connaître la signification littérale d'une énonciation contenant une expression de ce type implique toujours de savoir quel acte illocutionnaire a été effectivement accompli. En effet, si l'on accepte l'idée que tout acte locutionnaire n'entraîne pas nécessairement un acte illocutionnaire (comme dans le contexte pédagogique), la signification littérale d'un acte locutionnaire explicite l'acte illocutionnaire potentiellement accompli, plutôt que l'acte effectivement accompli. Nous remercions les rapporteurs de Philosophiques pour leur insistance sur ce point.

47. C'est à peu de choses près en ces termes que Sellars (I953) introduit le paradoxe auquel conduit la conception du langage comme pratique essentiellement régie par des règles. 
Revenons quelque peu en arrière sur la dimension normative de la signification telle qu'elle est introduite par Kripke, et notamment sur la manière dont ce dernier réfute la conception dispositionnaliste de la signification. Cette dernière conception, selon laquelle signifier revient à avoir une disposition verbale, ne rend pas compte du fait qu'utiliser un terme selon sa signification est un type d'acte où le locuteur manifeste une certaine maîtrise: la capacité à se conformer à une règle. Notre hypothèse est que signifier quelque chose lors d'une énonciation, c'est accomplir un acte locutionnaire, qui n'est pas seulement la manifestation d'une disposition à énoncer des phrases, mais aussi celle d'une certaine capacité à conformer son action à un standard, à une norme. Cela ne signifie pas qu'accomplir un acte locutionnaire implique d'avoir une certaine représentation mentale. Le locuteur peut avoir une certaine représentation, consciente ou implicite, qui coïncide avec son énonciation, mais cela ne suffit pas à hisser son énonciation au statut de locution, à faire de son acte l'instance d'une conformité intentionnelle à une règle: le locuteur doit, de plus, considérer son acte comme visant à la correction, comme relevant d'un genre d'acte qui peut être correct ou incorrect. Comme le formule Hannah Ginsborg ${ }^{48}$, le locuteur doit avoir une certaine "attitude normative " à l'égard de son acte, mais cette attitude normative ne peut consister en une représentation de la règle, sous peine de retomber dans le paradoxe wittgensteinien que nous avons présenté.

Supposons l'apprentissage d'une forme rudimentaire d'addition par un enfant ${ }^{49}$. L'enfant peut apprendre à écrire une suite de nombres en ajoutant « 2 » systématiquement: $2,4,6,8 \ldots$ À un certain stade de l'apprentissage, l'enfant est capable de continuer la suite correctement, mais cela n'implique pas qu'il soit capable de justifier sa réponse. L'enfant a une forme de conscience primitive de la correction de son acte, qui ne dépend pas de sa capacité à se représenter consciemment une règle. Encore une fois, si la capacité à se conformer à la règle suppose de se représenter la règle, alors cette capacité n'explique rien. Il s'agit plutôt ici d'un cas où le locuteur accomplit une action que l'on peut décrire adéquatement, pour reprendre l'analogie entre langage et jeu, comme un coup dans le cadre de la partie, mais où ce coup n'est ni le résultat d'une application consciente et explicite de la règle (comme c'est le cas généralement lorsque l'on joue à un jeu), ni simplement une action hasardeuse qui s'avère être conforme aux règles. C'est ce type d'état intermédiaire que Ginsborg appelle une «attitude normative primitive». L'attitude est normative au sens où l'acte est considéré comme correct, adéquat ou approprié, c'est-à-dire que l'acte ne peut être adéquatement décrit qu'au moyen d'un concept normatif, et en même temps

48. H. Ginsborg (20II).

49. Nous reprenons l'exemple de Ginsborg. 
la normativité en question est primitive au sens où elle «ne dépend pas de la conformité à une règle reconnue préalablement $t^{50}$ ».

Cette hypothèse soulève de nombreux problèmes que nous ne traiterons pas ici: en quoi consiste cette attitude normative? En particulier, implique-t-elle la possession du concept de correction? Et si c'est le cas, comment celui qui n'est pas encore un membre accompli de la communauté linguistique, comme l'enfant, peut-il déjà posséder ce concept? On peut, bien sûr, supposer que la capacité à avoir ce type d'attitude normative est innée, mais nous ne nous aventurerons pas dans cette direction. De façon beaucoup plus modeste, nous avancerons l'hypothèse que la normativité relève du concept d'acte locutionnaire, tout en restant neutre sur la question de savoir si tout locuteur doit nécessairement (en vertu de ce qu'est une énonciation douée de sens) instancier une telle attitude normative.

Pour introduire cette hypothèse, considérons un cas concret. Imaginons un enfant qui commence à parler. L'enfant commence à s'approprier des mots en les utilisant, mais pas nécessairement de la façon dont nous le faisons: l'enfant peut énoncer un mot pour s'amuser, pour entraîner sa capacité d'articulation, pour attirer l'attention, ou pour exprimer des émotions. Ces mots ont pour l'enfant une fonction qui n'est pas spécifiquement la leur, comme un livre peut servir à caler une table. Autrement dit, les mots n'ont pas la fonction qui leur est conventionnellement attribuée dans sa communauté linguistique. À quelles conditions l'enfant utilise-t-il intentionnellement un mot déterminé selon sa signification? L'enfant pourrait utiliser le mot «bain» chaque fois qu'il est dans la salle de bain, en manifestant un usage que nous serions tenté de qualifier de référentiel. Considérerionsnous, dans ce cas, que l'enfant connaît la signification de «bain» et fait un usage conforme à cette signification? L'enfant pourrait utiliser tout un tas de mots avec des fonctions différentes, lors d'énonciation que nous peinerions dans certains cas à interpréter, ou utiliser certains mots au hasard, dans des circonstances que nous jugerions appropriées, de sorte que nous serions tentés de lui attribuer la maîtrise d'un certain usage linguistique. En dépit de l'enthousiasme de ses parents, cela ne ferait pas de lui un locuteur, ni de son proto-langage un idiolecte. Nous supposons d'ordinaire que, pour que l'enfant manifeste une authentique compétence locutionnaire, et non simplement sententielle, ce dernier doit satisfaire certaines conditions, comme celle d'être dans la relation appropriée avec les mots qu'il énonce. Mais peut-être n'y a-t-il aucune propriété déterminée, aucun type d'état mental primitivement normatif, ou aucune disposition à utiliser correctement un terme dans des contextes suffisamment différents, que l'enfant devrait instancier ou manifester pour satisfaire cette condition. Ou peut-être que l'apprenti locuteur doit simplement être disposé à avoir une certaine 
attitude dans certaines circonstances, ou par moments. Mais dans quelle circonstance et à quel moment ?

L'ontogenèse de la compétence linguistique est certainement un processus graduel ${ }^{51}$, et il n'y a probablement aucun stade décisif où les balbutiements verbaux deviennent d'authentiques actes de langage (locutionnaires et illocutionnaires). Toutefois, notre concept de locution distingue entre l'énonciation arbitraire, mécanique ou tout simplement non signifiante et l'acte d'énoncer des mots en conformant son usage à certaines contraintes sémantiques, bien qu'il puisse exister des cas où la frontière entre ces deux types d'acte soit floue. L'idée est la suivante: lorsque nous disons d'un individu qu'il signifie quelque chose, au sens locutionnaire que nous avons donné à ce terme, nous le situons dans le cadre de notre pratique, nous l'incluons comme membre de notre communauté linguistique et nous lui attribuons une certaine capacité pratique et sociale. Cela signifie qu'il est un être sensible aux contraintes que la signification des termes qu'il utilise exercent sur son usage. Attribuer une signification littérale à l'énonciation d'un enfant, c'est-à-dire supposer que son énonciation possède une signification déterminée par la signification conventionnelle des expressions impliquées, c'est lui attribuer du même coup un statut de locuteur, ce qui est très différent du fait d'interpréter ce qu'il a voulu dire, ou son intention de communication $^{52}$. Être un locuteur implique d'être un participant dans une pratique où les actes sont susceptibles d'être critiqués, évalués ou corrigés à la lumière de certaines normes et standards. En vertu du fait qu'il est un participant de cette pratique essentiellement régulée, le locuteur est soumis à certaines obligations (sémantique) auquel il est, par définition, sensible.

On pourrait objecter que les concepts de locution ou d'acte locutionnaire ne jouent aucun rôle effectif dans l'apprentissage linguistique. Par opposition, on peut avancer que le concept de connaissance joue un rôle dans certaines pratiques épistémiques, comme l'enquête ou la délibération collective, notamment dans l'apprentissage des compétences nécessaires à ces pratiques. Mais est-ce le cas des concepts de locution ou d'acte locutionnaire? Tout d'abord, le terme d'acte locutionnaire est un terme technique, essentiellement familier des théoriciens des actes de langage, et il est clair que le terme n'est pas usité dans le cadre pédagogique de l'école ou du foyer familial. Mais cela ne signifie pas que le concept d'un acte où des mots sont énoncés selon leur signification n'ait pas un rôle à jouer. Le terme d'assertion est lui aussi restreint à un champ théorique, mais le concept d'un acte

51. Bloom 2000 insiste sur le fait qu'il n'y a pas, par exemple, d' "accélération " soudaine du vocabulaire. Il y a néanmoins un stade chez l'enfant (de 9 à I 2 mois) où celui-ci manifeste la capacité à comprendre que les autres ont des intentions de communication (Tomasello 2004).

52. Lorsque nous disons d'un enfant qui commence à parler qu'il a dit "papa ", nous voulons dire qu'il a énoncé "papa ", et non qu'il a utilisé "papa " selon sa signification conventionnelle, pour signifier PAPA, autrement dit pour accomplir un acte locutionnaire. 
de langage qui tend à la vérité et dont le contenu doit être connu du locuteur intervient dans des contextes tout à fait ordinaires (et non limités au cadre pédagogique), par exemple lorsqu'on critique quelqu'un qui asserte ce qu'il ne sait pas. Lorsqu'on rectifie ou critique un usage donné, on détourne l'attention qui est ordinairement accordée à l'acte illocutionnaire ou perlocutionnaire pour la recentrer sur la locution elle-même; ce faisant, on se demande si les mots ont été utilisés correctement, ce qui n'est autre que se demander si l'acte locutionnaire a été correctement accompli. Bien que l'attention portée sur la correction d'un usage soit, en un sens, secondaire relativement à la visée communicative du langage, où les significations et usages sont conçus comme des moyens pour déclarer, demander, ordonner, amener l'auditeur à faire ou croire quelque chose, le concept d'acte locutionnaire ou de locution correcte intervient de manière centrale lors de toute évaluation sémantique. Corriger le mauvais usage d'un enfant, évaluer les phrases d'un élève, c'est rappeler l'énonciateur à sa condition de locuteur.

\section{Conclusion}

Comment notre concept d'acte locutionnaire est-il lié au problème métaphysique de la nature de la signification? Notre stratégie a été de restreindre la question de la signification à la signification linguistique, c'est-à-dire à ce qu'est signifier au moyen d'un langage en principe public, laissant ainsi de côté la question de savoir comment un état mental peut avoir un contenu intentionnel, et ce en quoi consiste le fait d'avoir un certain concept doté d'un contenu déterminé. Nous avons proposé de traiter le concept d'acte locutionnaire comme central dans la pratique linguistique et de comprendre ce concept de façon normative, mais nous n'avons pas cherché à montrer que signifier, aussi bien dans le cas de la pensée que du langage, est nécessairement ou essentiellement un phénomène normatif. Autrement dit, nous n'avons pas cherché à montrer que la possibilité même de signifier quoi que ce soit suppose de prendre part à une pratique commune conventionnellement régulée. Un pas supplémentaire dans cette direction eût consisté à montrer que la nature de la signification est constituée (notamment) par de tels actes locutionnaires, une conception que l'on étiquette parfois sous l'appellation de "théorie de la signification comme usage ${ }^{53}$ ». C'est un pas que nous n'avons pas franchi. Par conséquent, dans notre perspective, la normativité ne constitue pas, comme le soutient Boghossian, une contrainte $a$ priori sur toute théorie de la signification, mais dépend au contraire d'une certaine conception de la signification dans le contexte de la pratique linguistique.

En outre, nous nous sommes contentés de faire une hypothèse conceptuelle. Cette hypothèse porte sur ce que signifie le fait de dire de quelqu'un

53. Paul Horwich (I998) défend une telle conception, mais rejette la thèse de la normativité de la signification telle que la comprend Kripke. 
qu'il a signifié quelque chose (au sens où il a accompli un acte locutionnaire, où le produit de son acte est adéquatement décrit comme une locution). L'idée sous-jacente est donc qu'isoler un certain sens de "signifier ", ou un certain concept de signification, impliqué dans la théorie de l'usage du langage (dont la théorie des actes de langage fait partie), permet de rendre compte de notre intuition selon laquelle «la signification est chargée de normes ", pour reprendre l'expression de Sellars. Il y a, semble-t-il, au moins deux réticences possibles face à de type de démarche. On peut nier la possibilité de parvenir à des vérités, ou du moins, des perspectives intéressantes, à partir d'une investigation du concept de signification ou de l'usage du terme "signification" (ou de termes apparentés). Bien qu'il y ait effectivement différents concepts de signification et de nombreux usages du terme, cela ne constitue pas une raison suffisante pour endosser un scepticisme radical quant à une approche conceptuelle ${ }^{54}$ de la notion, et surtout pour refuser une première clarification. Ce scepticisme est néanmoins utile pour rappeler à la prudence celui qui cherche à tirer des conclusions métaphysiques de cette approche. Cette dernière remarque nous mène directement au second type de réticence possible. On pourrait soutenir que l'hypothèse avancée ici laisse complètement en l'état la question de savoir si, oui ou non, il y a une forme de normativité essentielle dans le fait de signifier quelque chose. Nous pensons avoir en partie déjà répondu à cette objection. Il est clair que si l'on conçoit la tâche du théoricien de la signification dans une perspective exclusivement naturaliste, au sens d'une démarche qui vise la réduction des propriétés sémantiques à des propriétés naturelles (comme des fonctions biologiques), de telles remarques quant aux liens entre les concepts de signification, de norme et de locution sembleront alors largement tautologiques. Mais cela montre seulement que la portée de la clarification conceptuelle que nous avons esquissée ne prend tout son sens que dans le cadre plus large d'une théorie de la signification comme usage, telle qu'initiée par des auteurs comme Wittgenstein ou Wilfrid Sellars.

\section{Bibliographie}

Alston, William. Illocutionary Acts and Sentence Meaning, Ithaca, New York, Cornell University Press, 2000.

Austin, John. Quand dire, c'est faire, Paris, Seuil, 1970.

Baker, G. P., \& P. M. S. Hacker. Wittgenstein: Rules, Grammar and Necessity, Oxford, Wiley-Blackwell, 2009.

Blakemore, Diane. "Discourse Markers» dans Laurence R. Horn et Gregory Ward (dir.), Handbook of Pragmatics, Oxford, Blackwell, 2004.

Bloom, Paul. How Children Learn the Meaning of Words, Cambridge, MIT Press, 2000.

54. On peut, toutefois, être sceptique quant à l'analyse conceptuelle ou à la distinction apparentée entre l'analytique et le synthétique pour d'autres raisons. 
Boghossian, Paul. "The Rule-following Considerations", Mind, 98, I989, rééd. dans Content and justification, Oxford, Oxford University Press, 2009.

Brandom, Robert. Rendre explicite, I, Paris, Les Éditions du Cerf, 20 Iо.

Broome, John. Rationality through Reasoning, Oxford, Wiley-Blackwell, 2013.

Burgess, Alexis, et Brett Sherman (dir.), Metasemantics. New Essays on the Foundations of Meaning, Oxford, Oxford University Press, 2014.

Deacon, Terrence. The Symbolic Species. Co-Evolution of Language and the Brain, New York, W. W. Norton \& Company, I998.

Drestke, Fred. «Norms, History and the Mental», Royal Institute of Philosophy Supplements, 49, 2001, p. 87-104, rééd. dans Perception, Knowledge and Belief. Selected Essays, Cambridge, Cambridge University Press, 2000.

Engel, Pascal. «In Defense of Normativism about the Aim of Belief », dans Timothy Chan, The Aim of Belief, Oxford, Oxford University Press, 2013.

Foot, Philippa. "Morality as a System of Hypothetical Imperatives », The Philosophical Review, vol. 81, no 3, I972.

Gibbard, Alan. Normativity and Meaning, Oxford, Oxford University Press, 20I 2.

Ginsborg, Hannah. "Primitive Normativity and Skepticism about Rules", The Journal of Philosophy, 108, 5, 20I I.

Glüer, Kathryn, et Asa Wikforss. "Against Content Normativity ", Mind, I I 8, 2009.

—. "Meaning Normativism: Against the Simple Argument ", Organon F (Supplementary Issue), 22, 20I 5 .

Grice, Paul. Study in the Ways of Words, Harvard, Harvard University Press, I989.

Hattiangadi, Anandi. Oughts and Thoughts. Rule-Following and the Normativity of Content, Oxford, Oxford University Press, 2007.

- . "Some More Thoughts on Semantic Oughts: A Reply to Daniel Whiting", Analysis, 69, 2009.

Hazlett, Alan. A Luxury of the Understanding. On the Value of True Belief, Oxford, Oxford University Press, 2013.

Horwich, Paul. Meaning, Oxford, Oxford University Press, I999.

- Wittgenstein's Metaphilosophy, Oxford, Oxford University Press, 20 I I.

Iten, Corinne. Linguistic Meaning, Truth Conditions and Relevance. The Case of Concessives, Palgrave-Macmillan, 2005.

Kiesewetter, Benjamin. The Normativity of Rationality, Oxford, Oxford University Press, 2017.

Knight, Chris, Michael Studdert-Kennedy, et James R. Hurford (dir.), The Evolutionary Emergence of Language. Social Function and the Origins of Linguistic Form, Cambridge, Cambridge University Press, 2000.

Kripke, Saül. Règles et langage privé. Introduction au paradoxe de Wittgenstein, trad. T. Marchaisse, Paris, Seuil, I996.

Lewis, David. Convention, Cambridge, Harvard University Press, I969.

Millikan, Ruth Garrett. Language. A Biological Model, Oxford, Oxford University Press, 2005.

Raz, Joseph. Practical Reason and Norms, I ${ }^{\text {re }}$ éd. Hutchinson \& Co. I975, rééd. Oxford, Oxford University Press, I 999.

Récanati, François. "Pragmatics and Semantics», dans Laurence R. Horn et Gregory Ward (dir.), Handbook of Pragmatics, Oxford, Blackwell, 2004.

Searle, John. Speech Acts. An Essay in the Philosophy of Language, Cambridge, Cambridge University Press, I969. 
Searle, John, et Daniel Vanderveken. Foundations of Illocutionary Logic, Cambridge, Cambridge University Press, I982.

Sellars, Wilfrid. "Some Reflections on Language Games", Philosophy of Science, 2I, I954, p. 204-228, rééd. dans K. Sharp et R. Brandom, In the Spaces of Reasons, Selected Essays of Wilfrid Sellars, Cambridge, Harvard University Press, 2007.

Sperber, Dan, \& Deirdre Wilson. "Linguistic Form and Relevance ", Lingua, 90, I993, I-25, rééd. dans Meaning and Relevance, Cambridge, Cambridge University Press, 20I 2.

Steinberger, Florian. "The Normative Status of Logic», dans E. Zalta, Stanford Encyclopedia of Philosophy, 2016.

Tomasello, Michael. Constructing a Language, A Usage-based Theory of Language Acquisition, Cambridge, Harvard University Press, 2004.

Thomson, Judith Jarvis. Normativity, Chicago, Open Court, 2009.

Verheggen, Claudine. "Semantic Normativity and Naturalism», Logique \& Analyse, $2 \mathrm{I} 6,20 \mathrm{II}$.

Whiting, Daniel. "Is Meaning Fraught with Ought?", Pacific Philosophical Quarterly, 90, 2009.

—. "What Is the Normativity of Meaning? ", Inquiry, vol. 59, Iss. 3, 2016.

Wittgenstein, Ludwig. Recherches philosophiques, Paris, Gallimard, NRF, 2004.

Wright, Crispin. "Kripke's Account of the Argument against Private Language", The Journal of Philosophy, vol. 8I, no I2, p. 759-778, I984.

Zelaniec, Wojciech. "Remarks on The Ontology and The Normative Aspect of Constitutive Rules ", Etica \& Politica / Ethics \& Politics, XII, p. 407-425, 2010. 